\title{
Spontaneous Emulsification Via Phase Inversion At Ambient Temperature For Stable N-Octane-Aerosol-OT/Water Microemulsions
}

\author{
Kristina Simonova $^{1}$, Tatiana Samarina ${ }^{2}$, Akihide Hibara ${ }^{3}$, Mikhail Proskurnin ${ }^{1}$ \\ ${ }^{1}$ Lomonosov Moscow State University, Leninskie Gory 1, str. 3, GSP-1 Moscow, 119991, Russia. \\ kristina.1obko@gmail.com; proskurnin@gmail.com \\ ${ }^{2}$ Kajaani University of Applied Sciences, Ketunpolku 1, Kajaani, FI-87101, Finland \\ tatiana.samarina@kamk.fi \\ ${ }^{3}$ Institute of Multidisciplinary Research for Advanced Material \\ Tohoku University, 2-1-1 Katahira, Aoba-ku Sendai 980-8577, Japan \\ hibara@tohoku.ac.jp
}

\begin{abstract}
A reliable and straightforward way to prepare oil-in-water microemulsions based on AOT (Aerosol-OT, sodium 2ethylhexylsulfosuccinate) —n-octane-AOT/water or $\mathrm{NaCl}$ by a spontaneous-emulsification technique via dilution-triggered isothermal phase inversion in an inexpensive yet procurable way is demonstrated. The salt content adjusts the behavior and resulting microemulsions with long-term stability in the aqueous phase. Sudan I azo dye as a model solute is used to demonstrate variations in the resulting microemulsions and opportunities for preconcentration/separation or solute solubilization.
\end{abstract}

Keywords: spontaneous emulsification; microemulsion, solubilization, phase inversion, Sudan I

\section{Introduction}

Spontaneous emulsification (SE) with a surfactant is a reliable yet straightforward low-energy method for oil-in-water $(\mathrm{O} / \mathrm{W})$ microemulsion preparation, requiring no special equipment but simple mixing of constituents. SE has several advantages over high-energy microemulsification methods: it protects reactive species against severe conditions, does not require thermostatic conditions or co-surfactants, and in general needs lower surfactant amounts [1], [2]. The SE phenomenon has been a focus of attention for a long time in basic studies [3], [4] and diverse industrial applications such as pharmaceutical [5], cosmetic and food [6], [7], oil recovery [8], etc. Reviews on both micro- and nanoemulsions (which differ by stability and particle size) formed by SE may be found elsewhere [9]. A great effort has been made to implement SE for droplet-based pico- to nanoliter microfluidic systems [10]. However, at a macroscale, SE is not employed widely in applied chemistry since specific procedures for each system are needed due to variable mechanisms of SE. SE applications have recently been developed, e.g., for sample pretreatment interfaced with inductively coupled plasma atomic-emission [11] or atomicabsorption spectrometries [12]. Hence, reliable and straightforward ways of SE preparation are required for its coupling with various synthetic or analytical techniques. Among different SE procedures [13], we chose and implied a preparation method, where an isothermal phase-inversion process causes SE. It converts the initial system, a water-in-oil (W/O) nanoemulsion [14], to a phase that is supersaturated in oil, leading to the nucleation; the use of AOT (sodium 2-ethylhexylsulfosuccinate) provides reasonable control of the droplet size of the formed emulsion yet large microemulsion regions [15]. Three systems prepared at different salinities in the presence of an organic solute demonstrate the variety of the behavior and the properties of resulting microemulsions. Sudan I (1-phenylazo-2-naphthol) was used as a model solute to trace the possible influence of hydrophobic substances on the systems and show preconcentration/separation or solute solubilization.

\section{Materials and methods}

\subsection{Materials}

Sodium bis(2-ethylhexyl)sulfosuccinate/Aerosol-OT/AOT (98\%, Sigma Aldrich, USA), sodium chloride (analytical grade, Reakhim, Russia), n-octane (Vekton, Russia), Sudan I, 1-phenylazo-2-naphthol (analytical grade, Reakhim, Russia) were used throughout. Formazin was used as a turbidity calibration standard (certified reference material, 4000 NTU, Ltd. Saint-Petersburg). Reagents were used as received. All the aqueous solutions were prepared with water from a Milli Q water purification system (Millipore, France; specific resistance, $18.2 \mathrm{M} \Omega \times \mathrm{cm}$; total ion amount, $<0.2 \mathrm{ppb}$; TOC, below $10 \mathrm{ppb}$ ). 


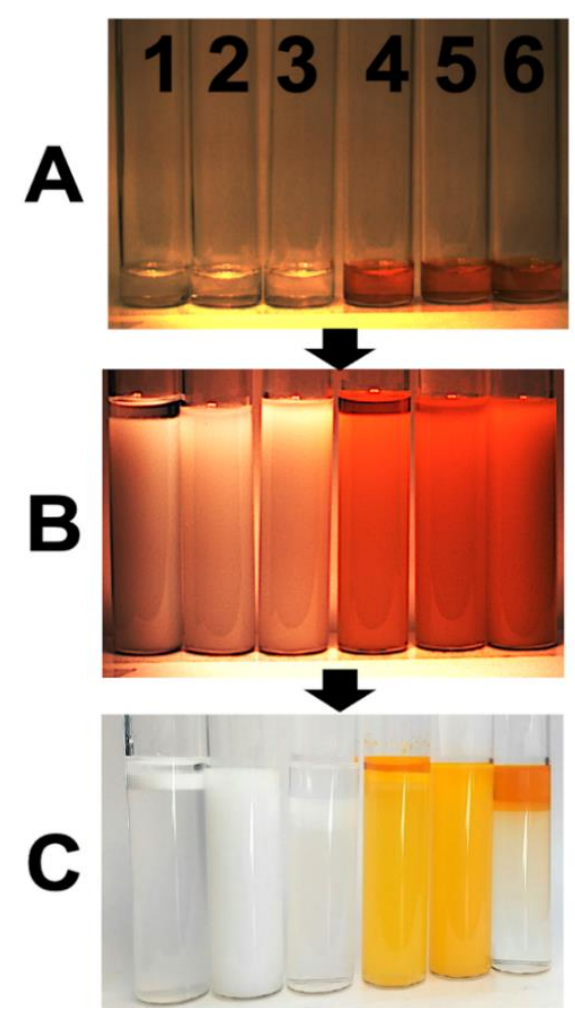

\subsection{Equipment}

All nano- and microemulsions were prepared in screw-top glass vials to prevent composition changes. An OHAUS Discovery analytical balance (Switzerland; precision, $\pm 0.1 \mu \mathrm{g}$ ) was used. Sartorius-Biohit Proline singlechannel pipettes (Finland) were used for liquid constituents. Absorption spectra were measured in 300-800 nm using an Agilent Cary 60 spectrophotometer (USA) in quartz cells (path lengths, $1 \mathrm{~cm}$ ) at room temperature. The turbidity values were determined at $545 \mathrm{~nm}$ with a Varian Cary 4000 (USA) spectrophotometer. Mean hydrodynamic diameters of oil microdroplets were determined by dynamic light scattering (DLS) at a fixed angle $\left(90^{\circ}\right)$ in a thermostatic cell with a Brookhaven Zeta Plus instrument (USA), the measurement error is $6 \%$. The manufacturer software was used to calculate the volume and number-averaged diameters of droplets; the weighting function was generated by autocorrelator and cumulative distribution function.

\subsection{Emulsification procedure}

To prepare octane/AOT/water $(\mathrm{NaCl})$ initial W/O nanoemulsions with weight $\%$ ratios of 70/20/10, respectively, $0.30 \mathrm{~g}$ AOT is dissolved in $1.05 \mathrm{~g}$ of octane, then $0.15 \mathrm{~g}$ of deionized water (DI) or $\mathrm{NaCl}$ solution $(0.2$ or $0.4 \% \mathrm{wt}$.) is added to the organic phase resulting in transparent $\mathrm{W} / \mathrm{O}$ nanoemulsions (Fig. 1 , A, vials 1-3). For vials 4-6, the procedure was similar, but $0.30 \mathrm{~g}$ AOT is dissolved in $1.05 \mathrm{~g}$ of octane contained $2 \mathrm{mM}$ of Sudan I. Next, the obtained W/O nanoemulsion is diluted with $18 \mathrm{~mL}$ of $\mathrm{DI}$ or $\mathrm{NaCl}$ solution $(0.2 \%$ wt. or

Fig. 1: Phase behavior of microemulsions prepared by diluting initial W/O nanoemulsions of water-NaCl/n-octane-AOT of 10 : 70 : 20 wt.\% for different salinities of the aqueous phase. Time after SE: A: initial W/O microemulsions; B: after dilution and SE; and C: 5 days after dilution and SE. Vials 1-3 have no additional components, in vials 4-6 Sudan I azo dye solution in octane was used under the same conditions. From left to right for vials 1-3: system \#1 (diluent DI), system \#2 (diluent $0.2 \% \mathrm{NaCl}$ ) and system \#3 (diluent $0.4 \% \mathrm{NaCl}$ ). The numeration of vials from 1 to 6 is the same for moments $\mathrm{A}, \mathrm{B}, \mathrm{C}$.

$0.4 \%$ wt.) in a $20 \mathrm{~mL}$ screw-top glass vial at $25 \pm 5^{\circ} \mathrm{C}$, causing phase inversion and spontaneous formation of $\mathrm{O} / \mathrm{W}$ microemulsions (Fig. 1, B). The final AOT concentration was $1.5 \% \mathrm{wt}$. All systems were prepared in triplicate to gain statistical data via visual control or spectrometric and DLS measurements.

\section{Results and discussion}

It was shown [16], [17] that significantly different behavior and properties of resulting microemulsions can be tuned by the composition of the aqueous phase, e.g., the salinity of the initial nanoemulsion and diluents used to invert the phase. According to Winsor classification of emulsions (types I-IV), systems at three salinities were selected to form emulsions of different Winsor types [18]:

- Winsor I, no salt added, system \#1, vials 1 and 4,

- Winsor IV, $0.2 \%$ wt. $\mathrm{NaCl}$, system \#2 vials 2 and 5 ,

- Winsor II, $0.4 \%$ wt. $\mathrm{NaCl}$, system \#3, vials 3 and 6.

Nanoemulsions n-octane-AOT/water and n-octane-AOT/water-NaCl of $70: 20: 10 \mathrm{wt} . \%$, served as initial W/O systems, were prepared. The preparation of all selected initial W/O nanoemulsions results in transparent, homogeneous, and indistinguishable liquids (Fig. 1, A, vials 1-6). Shortly after that, initial W/O nanoemulsions were diluted with DI (no salt added, system \#1) or $\mathrm{NaCl}$ solutions $(0.2 \% \mathrm{wt}$. or $0.4 \% \mathrm{wt}$, system \#2 or \#3, resp.), causing phase inversion and spontaneous formation of $\mathrm{O} / \mathrm{W}$ microemulsions. Immediately after SE, the systems behave differently (Fig. 1, B, vials 1-6). Therefore, system \#1 has an immediate separation between the oil and microemulsion phases, while systems $\# 2$ and \#3 are homogeneous and almost immersed.

The systems were prepared several times to study the reproducibility of the preparation approaches. Parameters of the systems were monitored by turbidimetry and DLS. A simple forced single-exponent fitting (cumulant) scheme of 
DLS data treatment was used to find the mean size (by intensity), and a relative polydispersity index (PDI, ISO 22412:2017) was used for overall polydispersity (the normalized second cumulant) interpretation of the resulting microemulsions (Fig. 2).
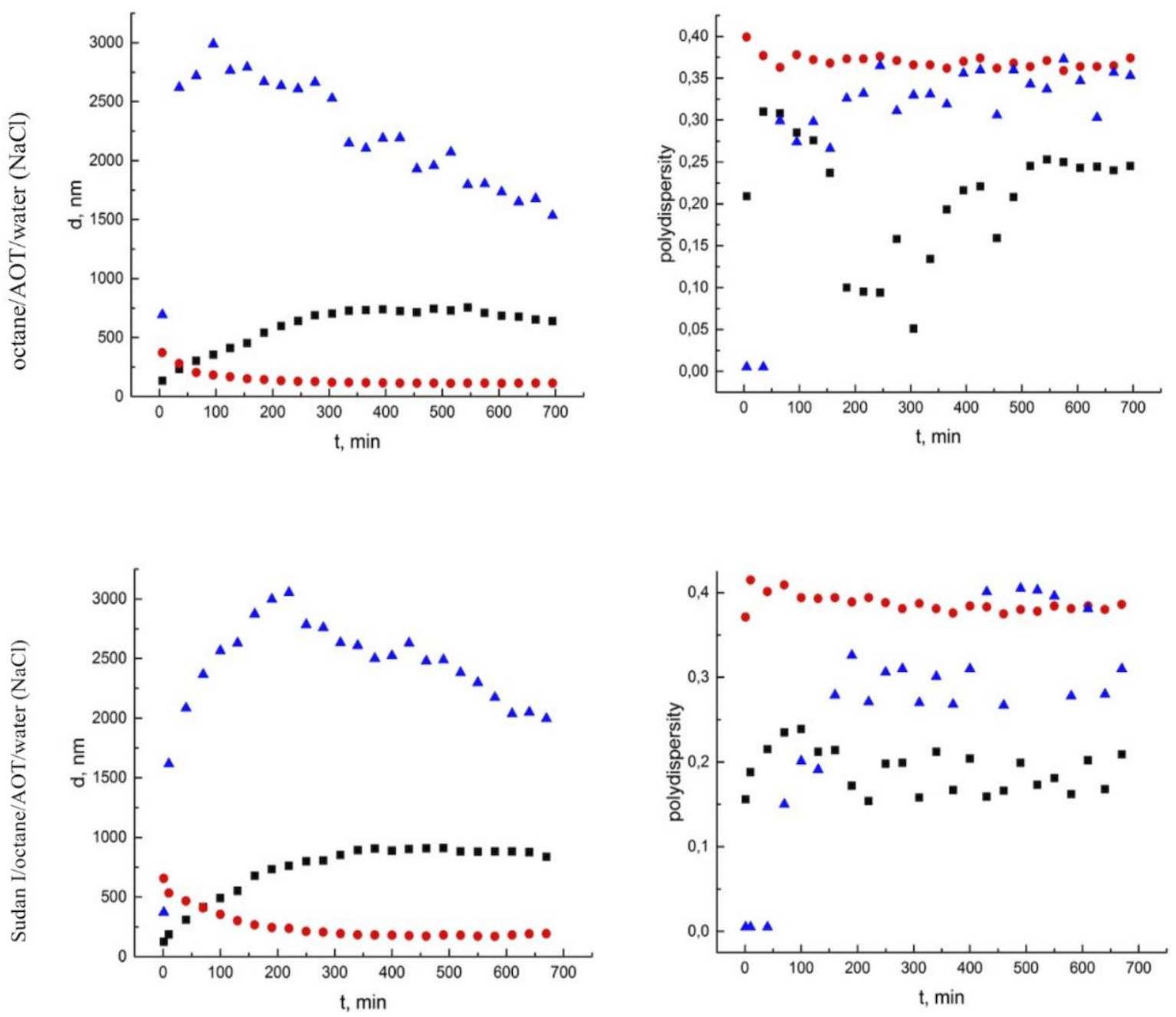

Fig. 2: Octane droplet size (left) and polydispersity (right) in microemulsions formed by diluting initial W/O nanoemulsions with: deionized water, $\square$ (system \#1); $0.2 \% \mathrm{NaCl}, \bullet$ (system \#2); and $0.4 \% \mathrm{NaCl}, \Delta$ (system \#3); without (upper) and with Sudan I (lower).

If the initial W/O nanoemulsion was diluted with DI (system \#1), droplet size 1-5 min after the contact was below the DLS limit of detection (less than $5 \mathrm{~nm}$ ). They grew for $5 \mathrm{~h}$ to $c a .1 \mu \mathrm{m}$ and did not change much for another $7 \mathrm{~h}$. Triplicate preparation and measurement of system \#1 resulted in an average diameter of $870 \pm 130 \mathrm{~nm}$ for both vials 1 and 4 (without and with Sudan I, resp.) in $600 \mathrm{~min}$ after SE. System \#1 has polydispersity altering with growing microdroplets. Wherein Sudan I (system \#1, Fig.1, C4) probably stabilized the distribution, and micrometer-sized particles $(890 \pm 70 \mathrm{~nm})$ arranged a system with PDI of 0.2 (Fig. 2, lower). The stability of the systems was also estimated visually and by the UV-vis method. In system \#1, the model solute distribution between phases was reproducible but complicated and time-dependent during the first $5 \mathrm{~h}$. Thus, it is not beneficial to use SE by phase inversion with water to further analyze hydrophobic compounds, but it may be used to form stable, transparent microemulsion after 5 hours of SE without extra energy input (Fig.1, C4).

System \#2 resulted in an average diameter of $140 \pm 40 \mathrm{~nm}$ for vials 2 and 5 (without and with Sudan I, resp.) and 600 min after SE. System \#2 with a $0.2 \% \mathrm{NaCl}$ solution (Fig. 2, vials 2 and 5) show significantly different behavior from systems $\# 1$ and \#3. Here, octane drop size decreased from 650 to $100 \mathrm{~nm}$ during the first $2 \mathrm{~h}$; the droplet sizes became stable for 10 h. For system \#2 with Sudan I (Fig.1, C, vial 5), the PDI was high (0.4) and constant (Fig. 2, lower) probably due to the small droplet size at the beginning and further shrinkage after SE, so the aggregation did not occur. By turbidimetric measurement, 
system \#2 was stable for 5 days, and visually this system did not collapse even after 5 months. This stability could be explained through the microdrop structure. Kini et al. [16] have shown that diameters over $c a .100 \mathrm{~nm}$, there was enough AOT for an initial monolayer and at least an extra bilayer for an incipient lamellar phase coating. A cloudy containing the solubilized Sudan I dye was obtained (Fig.1, C, vial 5). The final diameter of dye-containing droplets was higher than without the dye $(290 \pm 70 \mathrm{~nm})$, but no creaming was observed.

For system \#3 (Fig. 2), droplets grew up to $3 \mu \mathrm{m}$ at high rates for $2 \mathrm{~h}$, though in $10 \mathrm{~h}$ after SE, it resulted in an average diameter of $1900 \pm 500 \mathrm{~nm}$ for both vials 3 and 6 (without and with Sudan I, resp.). System \#3 for the first hour may be treated as monodisperse since PDI was below 0.1 according to ISO 22412:2017. Next, PDI increases, which may be attributed to aggregation/agglomeration processes. This effect may also be accounted for Ostwald ripening because the curve has a leap (after $60 \mathrm{~min}$ ) independent of the presence of hydrophobic compounds. Subsequently, the droplet size decreased (Fig. 2). It may result from a combination of coalescence of internal aqueous droplets (or with the external aqueous phase), mass transport of water from internal droplets to the external phase, and the breakup of larger oil drops due to convection inside oil droplets for system \#3. As was proven in [16] with optical microscopy under similar conditions, the dilution with $0.4 \% \mathrm{NaCl}$ favors oil-continuous microemulsions forming a W/O/W multiple emulsion. This situation may happen because a higher salinity promotes the stability of W/O emulsions. The organic phase of droplets is a W/O microemulsion; its swollen inverse micelles provide a mechanism for mass transport of water from the internal droplets to the external aqueous phase; the former has a higher pressure, thus, a higher chemical potential of water owing to droplet curvature. Both coalescence and mass transfer in multiple emulsions are discussed elsewhere [19]. For system \#3, a complete separation of Sudan I in the upper phase was observed. The concentration in the $\mathrm{W} / \mathrm{O}$ phase was carried out spectrophotometrically, and the enrichment factor was 1.2.

\section{Conclusions}

A low-cost and reliable method for preparing oil-in-water microemulsions, n-octane-AOT/water and n-octane$\mathrm{AOT} /$ water- $\mathrm{NaCl}$ in the presence of hydrophobic solute, by a spontaneous emulsification technique is demonstrated. The proposed approach does not require any intensive stirring or extra work input, and microemulsions with different properties occurred spontaneously by the phase inversion process. Properties of microemulsions can be reached by adjusting the composition of the aqueous phase - the salinity - of initial W/O emulsions and the diluents. Long-term stability of microemulsions gives the principal possibility to further separate or analyze an organic phase with a hydrophobic analyte and an $\mathrm{O} / \mathrm{W}$ microemulsion. These microemulsions are promising examples for developing sample pretreatment, preconcentration, or separation systems.

\section{Acknowledgments}

This research was performed according to the Development program of the Interdisciplinary Scientific and Educational School of Lomonosov Moscow State University, «The future of the planet and global environmental change».

We thank Dr. Natalya M. Zadymova (Lomonosov Moscow State University) for advice and Dr. Andrey V. Sybachin (Lomonosov Moscow State University) for the assistance in scattering measurements.

\section{References}

[1] J. C. López-Montilla, P. E. Herrera-Morales, S. Pandey, and D. O. Shah, 'Spontaneous Emulsification: Mechanisms, Physicochemical Aspects, Modeling, and Applications', Journal of Dispersion Science and Technology, vol. 23, no. 13, pp. 219-268, Jan. 2002, doi: 10.1080/01932690208984202.

[2] S. Shi, Y. Wang, L. Wang, Y. Jin, T. Wang, and J. Wang, 'Potential of Spontaneous Emulsification Flooding for Enhancing Oil Recovery in High-Temperature and High-Salinity Oil Reservoir', Journal of Dispersion Science and Technology, vol. 36, no. 5, pp. 660-669, May 2015, doi: 10.1080/01932691.2014.905954.

[3] C. A. Miller, 'Spontaneous Emulsification Produced by Diffusion - A Review', Colloids and Surfaces, vol. 29, no. 1, pp. 89-102, Jan. 1988, doi: 10.1016/0166-6622(88)80173-2.

[4] C. Solans, D. Morales, and M. Homs, 'Spontaneous emulsification', Current Opinion in Colloid \& Interface Science, vol. 22, pp. 88-93, Apr. 2016, doi: 10.1016/j.cocis.2016.03.002. 
[5] K. Bouchemal, S. Briançon, E. Perrier, and H. Fessi, 'Nano-emulsion formulation using spontaneous emulsification: solvent, oil and surfactant optimisation', International Journal of Pharmaceutics, vol. 280, no. 1, pp. 241-251, Aug. 2004, doi: 10.1016/j.ijpharm.2004.05.016.

[6] J. S. Komaiko and D. J. McClements, 'Formation of Food-Grade Nanoemulsions Using Low-Energy Preparation Methods: A Review of Available Methods', Comprehensive Reviews in Food Science and Food Safety, vol. 15, no. 2, pp. 331-352, 2016, doi: https://doi.org/10.1111/1541-4337.12189.

[7] H. D. Silva, M. Â. Cerqueira, and A. A. Vicente, 'Nanoemulsions for Food Applications: Development and Characterization', Food Bioprocess Technol, vol. 5, no. 3, pp. 854-867, Apr. 2012, doi: 10.1007/s11947-011-0683-7.

[8] Z. Li et al., 'Advances of spontaneous emulsification and its important applications in enhanced oil recovery process', Advances in Colloid and Interface Science, vol. 277, p. 102119, Mar. 2020, doi: 10.1016/j.cis.2020.102119.

[9] D. J. McClements, 'Nanoemulsions versus microemulsions: terminology, differences, and similarities', Soft Matter, vol. 8, no. 6, pp. 1719-1729, Jan. 2012, doi: 10.1039/C2SM06903B.

[10]M. Fukuyama and A. Hibara, 'Microfluidic Selective Concentration of Microdroplet Contents by Spontaneous Emulsification', Anal. Chem., vol. 87, no. 7, pp. 3562-3565, Apr. 2015, doi: 10.1021/acs.analchem.5b00155.

[11]A. N. Anthemidis, V. Arvanitidis, and J. A. Stratis, 'On-line emulsion formation and multi-element analysis of edible oils by inductively coupled plasma atomic emission spectrometry', Analytica Chimica Acta, vol. 537, no. 1, pp. 271278, Apr. 2005, doi: 10.1016/j.aca.2005.01.035.

[12]C. E. R. de Paula, G. F. B. Cruz, C. M. S. P. Rezende, and R. J. Cassella, 'Determination of Cr and Mn in moisturizing creams by graphite furnace atomic absorption spectrometry through direct introduction of the samples in the form of emulsions', Microchemical Journal, vol. 127, pp. 1-6, Jul. 2016, doi: 10.1016/j.microc.2016.01.017.

[13]C. E. Mora-Huertas, H. Fessi, and A. Elaissari, 'Influence of process and formulation parameters on the formation of submicron particles by solvent displacement and emulsification-diffusion methods: Critical comparison', Advances in Colloid and Interface Science, vol. 163, no. 2, pp. 90-122, Apr. 2011, doi: 10.1016/j.cis.2011.02.005.

[14]A. Rahdar and M. Almasi-Kashi, 'Dynamic and spectroscopic studies of nano-micelles comprising dye in water/ dioctyl sodium sulfosuccinate /decane droplet microemulsion at constant water content', Journal of Molecular Structure, vol. 1128, pp. 257-262, Jan. 2017, doi: 10.1016/j.molstruc.2016.08.076.

[15]T. Nishimi and C. A. Miller, 'Spontaneous Emulsification of Oil in Aerosol-OT/Water/Hydrocarbon Systems', Langmuir, vol. 16, no. 24, pp. 9233-9241, Nov. 2000, doi: 10.1021/la0006521.

[16]G. C. Kini, S. L. Biswal, M. S. Wong, and C. A. Miller, 'Characteristics of spontaneously formed nanoemulsions in octane/AOT/brine systems', Journal of Colloid and Interface Science, vol. 385, no. 1, pp. 111-121, Nov. 2012, doi: 10.1016/j.jcis.2012.07.041.

[17]M. K. Zabar, C. V. Nguyen, and C. M. Phan, 'Quantifying the influence of salinity on spontaneous emulsification of hydrocarbons', Colloids and Surfaces A: Physicochemical and Engineering Aspects, vol. 588, p. 124376, Mar. 2020, doi: 10.1016/j.colsurfa.2019.124376.

[18]P. A. Winsor, 'Hydrotropy, solubilisation and related emulsification processes', Trans. Faraday Soc., vol. 44, no. 0, pp. 376-398, Jan. 1948, doi: 10.1039/TF9484400376.

[19]A. Y. Khan, S. Talegaonkar, Z. Iqbal, F. J. Ahmed, and R. K. Khar, 'Multiple emulsions: an overview', Curr Drug Deliv, vol. 3, no. 4, pp. 429-443, Oct. 2006, doi: 10.2174/156720106778559056. 